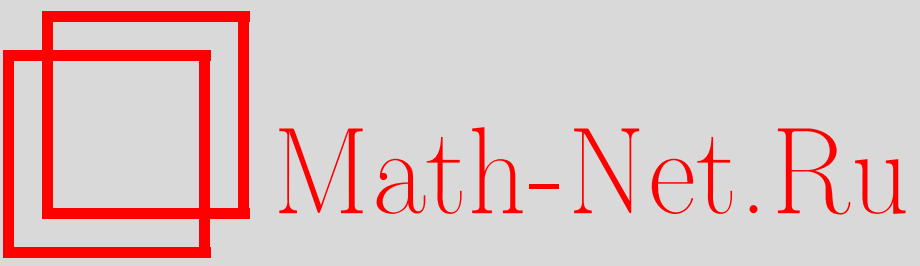

Ю. А. Кашлев, Термализация каскадов смещений в твердых телах и модель теплового пика, ТМФ, 2002, том 130, номер 1, 131-144

DOI: https://doi.org/10.4213/tmf295

Использование Общероссийского математического портала Math-Net.Ru подразумевает, что вы прочитали и согласны с пользовательским соглашением

http://www . mathnet.ru/rus/agreement

Параметры загрузки:

IP : 54.80 .73 .141

26 апреля 2023 г., $16: 36: 51$ 
ТЕОРЕТИЧЕСКАЯ

И МАТЕМАТИЧЕСКАЯ

ФИЗИКА

Том 130, № 1

январь, 2002

(C) 2002 г.

Ю. А. Кашлев*

\section{ТЕРМАЛИЗАЦИЯ КАСКАДОВ СМЕЩЕНИЙ В ТВЕР ДЫХ ТЕЛАХ И МОДЕЛЬ ТЕПЛОВОГО ПИКА}

Методами неравновесной статистической термодинамики исследована термализация низкоэнергетических каскадов смещений в твердых телах. С учетом фононного рассеяния на выбитых атомах и термической рекомбинации дефектов рассмотрена временна́я эволюция квазитемпературы каскадных частиц. На основе полученного временно́го хода квазитемпературы изучен активированный процесс - термическое распыление в области теплового пика.

\section{1. ВВЕДЕНИЕ}

Вопросы термодинамики каскадных процессов в твердых телах рассматривались в работах [1], [2]. В них исследованы ранние этапы каскадообразования - каскадный (характерное время $\sim 10^{-13} \mathrm{c}$ ) и релаксационньй (с характерным временем $\sim 10^{-12} \mathrm{c}$ ). Эти этапы завершаются установлением квазиравновесия в подсистеме первично выбитых атомов (ПВА) и вторичных атомов отдачи, т.е. в подсистеме каскадных частиц (КЧ). Поскольку квазиравновесие далеко от полного термодинамического равновесия системы, то вслед за ранними этапами идет заключительный этап - термализация. На этом этапе происходит переход системы через последовательность состояний текушего квазиравновесия, каждое из которых определяется значениями термодинамических параметров в данный момент времени. В результате состояние системы зависит от времени только через медленно меняюшиеся термодинамические параметры $F_{i m}(t)$. Более грубый подход использовался в работах [3]-[7], посвященных термализации позитронов. Что же касается термализации КЧ, то ее изучение на основе кинетических уравнений для параметров $F_{i m}(t)$ представляется достаточно новым направлением в теории каскадообразования. К этому следует добавить, что в данной работе основным механизмом отвода тепла из каскадной зоны считается диссипация энергии за счет атом-атомных столкновений. Механизмы, связанные с электронным рассеянием, дают малый вклад [8] и не рассматриваются в дальнейшем.

\footnotetext{
* Институт металлургии и материаловедения им. А. А. Байкова РАН, Москва, Россия
} 


\section{2. КИНЕТИЧЕСКИЕ УРАВНЕНИЯ ДЛЯ ТЕРМОДИНАМИЧЕСКИХ ПАРАМЕТРОВ НА СТАДИИ ТЕРМАЛИЗАЦИИ КАСКАДА. ПОТОК ЭНЕРГИИ}

Система, состоящая из кристалла (термостат) и подсистемы КЧ, допускает сокрашенное описание с помошью двух переменных: $P_{1 i}=H_{i}$ - гамильтониан и $P_{2 i}=N_{i}$ число частиц $(i=1,2)$. Неравновесное состояние такой системы описывается совокупностью средних значений переменных $P_{m i}$, а функционал энтропии - соотношением

$$
S(t)=-\operatorname{Sp}\left\{\rho_{l}(t) \ln \rho_{l}(t)\right\} .
$$

Здесь

$$
\rho_{l}(t)=\exp \left\{-\Phi+\sum_{m i} P_{m i} F_{i m}(t)\right\}
$$

- неравновесный статистический функционал, $\Phi$ - функционал Масье-Планка. Функции $F_{i m}(t)$ выбираются таким образом, чтобы истинные средние $\left\langle P_{m i}\right\rangle$ были равны квазиравновесным средним $\left\langle P_{m i}\right\rangle_{l}^{t}=\operatorname{Sp}\left\{\rho_{l}(t) P_{m i}\right\}$. Тогда они имеют следуюший смысл: $1 / F_{11}=1 / \beta_{1}=T_{1}$ - температура термостата; $1 / F_{21}(t)=1 / \beta_{2}(t)$ - квазитемпература $\mathrm{KЧ} ; F_{12}=-F_{11} \mu_{1}, F_{22}(t)=-F_{21}(t) \mu_{2}(t) ; \mu_{2}(t)$ - квазихимический потенциал $\mathrm{K} Ч$, $\mu_{1}$ - химический потенциал термостата.

После установления квазиравновесия в подсистеме КЧ дальнейшая эволюция термодинамических параметров определяется уравнением [9]

$$
\dot{F}_{i m}(t)=\sum_{n j} \frac{\delta^{2} S(t)}{\delta\left\langle P_{m i}\right\rangle_{l}^{t} \delta\left\langle P_{n j}\right\rangle_{l}^{t}} J_{n j}(t) .
$$

Для потоков $J_{n j}(t)$ справедливы соотношения

$$
J_{n j}(t)=\left\langle\dot{P}_{n j}\right\rangle_{l}^{t}=-i \operatorname{Sp}\left\{\rho_{l}(t)\left[P_{n j}, H\right]\right\},
$$

где $H$ - полньй гамильтониан системы. Здесь и далее используется система единиц, в которой $\hbar=k_{\mathrm{B}}=1$. Функциональную производную в (1) нетрудно связать с корреляционной функцией

$$
\left(P_{m i}, P_{j i}\right)^{t}=\operatorname{Sp}\left\{\rho_{l}(t) P_{m i} P_{j i}\right\},
$$

для этого достаточно воспользоваться термодинамическими равенствами

$$
\begin{aligned}
& \frac{\delta F_{i m}(t)}{\delta\left\langle P_{n j}\right\rangle_{l}^{t}}=\frac{\delta^{2} S(t)}{\delta\left\langle P_{n j}\right\rangle_{l}^{t} \delta\left\langle P_{m i}\right\rangle_{l}^{t}}=\frac{\delta F_{j n}(t)}{\delta\left\langle P_{m i}\right\rangle_{l}^{t}}, \\
& \frac{\delta\left\langle P_{m i}\right\rangle_{l}^{t}}{\delta F_{j n}(t)}=-\left(P_{n j}, P_{m i}\right)^{t}=\frac{\delta\left\langle P_{n j}\right\rangle_{l}^{t}}{\delta F_{i m}(t)} .
\end{aligned}
$$

Используя эти равенства и уравнение (1), а также принимая во внимание условие термодинамического равновесия термостата $\dot{\beta}_{1}=0$, находим

$$
\begin{aligned}
& \left(H_{2}, H_{2}\right)^{t} \dot{\beta}_{2}(t)-\left(H_{2}, N_{2}\right)^{t}\left\{\dot{\beta}_{2}(t) \mu_{2}(t)+\beta_{2}(t) \dot{\mu}_{2}(t)\right\}=-J_{12}(t), \\
& \left(N_{2}, H_{2}\right)^{t} \dot{\beta}_{2}(t)-\left(N_{2}, N_{2}\right)^{t}\left\{\dot{\beta}_{2}(t) \mu_{2}(t)+\beta_{2}(t) \dot{\mu}_{2}(t)\right\}=-J_{22}(t) .
\end{aligned}
$$


Воспользовавшись этой системой, находим искомое кинетическое уравнение для квазитемпературы подсистемы КЧ,

$$
\left\{\left(H_{2}, H_{2}\right)^{t}-\frac{\left(N_{2}, H_{2}\right)^{t}\left(H_{2}, N_{2}\right)^{t}}{\left(N_{2}, N_{2}\right)^{t}}\right\} \dot{\beta}_{2}(t)=-J_{12}(t)+J_{22}(t) \frac{\left(H_{2}, N_{2}\right)^{t}}{\left(N_{2}, N_{2}\right)^{t}} .
$$

Усредняя диссипативную функцию [1], получаем общий вид потока энергии $J_{12}(t)$ из соотношения (2),

$$
J_{12}(t)=-\int d \varepsilon \tilde{p}\left(\beta_{2}(t), \varepsilon\right) \tilde{L}_{\dot{H}_{2} \dot{H}_{2}}(\varepsilon)\left\{\beta_{1}-\beta_{2}(t)\right\}
$$

Здесь

$$
\tilde{L}_{\dot{H}_{2} \dot{H}_{2}}(\varepsilon)=\frac{1}{2} \int d \omega \omega^{2} \int d \mathbf{q} \frac{1}{2 \pi^{2}}|V(q)|^{2} \beta_{1} \omega S^{(\mathrm{ph})}(\mathbf{q}, \omega) \delta((\mathbf{q v})-\omega)
$$

- коэффициент переноса, входящий в линейное соотношение между потоком энергии и термодинамической силой [1], $V(q)$ - фурье-компонента потенциала взаимодействия КЧ с колебаниями решетки, $\mathbf{v}-$ скорость КЧ,

$$
\tilde{p}\left(\beta_{2}(t), \varepsilon\right)=g(\varepsilon) n^{(2)}\left(\beta_{2}(t), \varepsilon\right)
$$

- энергетическое распределение КЧ, $g(\varepsilon)$ - плотность числа состояний КЧ, $n^{(2)}\left(\beta_{2}(t), \varepsilon\right)=\lambda \exp \left\{-\beta_{2}(t) \varepsilon\right\}$. Динамический формфактор, вошедший в (3б), определим с помошью стандартного выражения

$$
\begin{gathered}
S^{(\mathrm{ph})}(\mathbf{q}, \omega)=\frac{1}{2 \pi} \int_{0}^{\infty} d t \exp (i \omega t)\left\langle\rho_{-\mathbf{q}}^{(i)} \rho_{\mathbf{q}}^{(i)}(t)\right\rangle_{0}, \\
\left\langle\rho_{-\mathbf{q}}^{(i)} \rho_{\mathbf{q}}^{(i)}(t)\right\rangle_{0}=\exp (-2 \widetilde{W}) \sum_{\mathbf{R}} \exp (-i \mathbf{q} \mathbf{R})\langle(\mathbf{q u}(0, t))(\mathbf{q u}(\mathbf{R}, t))\rangle_{0} .
\end{gathered}
$$

В формулах (4) $\rho_{\mathbf{q}}^{(i)}(t)$ - фурье-компонента плотности атомов решетки, $\mathbf{u}(\mathbf{R}, t)$ - смешения атомов из положения равновесия $\mathbf{R}, \exp (-2 \widetilde{W})$ - фактор Дебая-Валлера, $\langle\ldots\rangle_{0}-$ усреднение по равновесному состоянию.

Теперь преобразуем коэффициент переноса (3б). А именно, вьполним интегрирование по угловым переменным вектора q. Получаем

$$
\tilde{L}_{\dot{H}_{2} \dot{H}_{2}}(\varepsilon)=\frac{1}{2 \pi^{2}} v \beta_{1} \int d \omega \omega^{2} \int d q q^{3}|V(q)|^{2} S^{(\mathrm{ph})}(q, \omega)\left|\int_{0}^{\infty} d t^{\prime} e^{-i \omega t^{\prime}} j_{1}\left(q v t^{\prime}\right)\right|,
$$

где $j_{1}(x)$ - цилиндрическая функция Бесселя. Затем проинтегрируем в $(5)$ по $d t$, а также по $d \omega$, предварительно разложив (4) по степеням $u$. В результате кинетический коэффициент (3б) с учетом однофононных (член, пропорциональный $u$ ) и двухфононных (член, пропорциональный $u^{2}$ ) процессов рассеяния может быть представлен следующим обра30м:

$$
\begin{gathered}
\tilde{L}_{\dot{H}_{2} \dot{H}_{2}}(\varepsilon)=\varepsilon \widetilde{\Phi}, \quad \widetilde{\Phi}=\widetilde{\Phi}^{(1)}+\widetilde{\Phi}^{(2)}, \\
\widetilde{\Phi}^{(1)}=\frac{1}{3 \pi^{2}} \frac{1}{M} \widetilde{\Omega}_{a} \int d q q^{6}\left(2 M \omega_{q}\right)^{-1}|V(q)|^{2} \beta_{1} \eta\left(\omega_{q}\right), \\
\widetilde{\Phi}^{(2)}=\frac{4}{3 \pi^{2}} \frac{1}{M} \widetilde{\Omega}_{a} \int d q q^{6}\left[\frac{1}{\omega_{q}^{3}}|V(q)|^{2}\right]^{2} \frac{1}{M} \int_{0}^{\Delta_{q}} d \widetilde{\omega} \widetilde{\omega}\left(\Delta_{q}-\widetilde{\omega}\right) \beta_{1} \eta(\widetilde{\omega}) \eta\left(\Delta_{q}-\widetilde{\omega}\right) .
\end{gathered}
$$


В (6) введены следующие обозначения: $M$ - масса атома решетки, $\omega_{q}$ - энергия колебательной моды, $\widetilde{\Omega}$ - объем, $\Delta_{q}$ - энергия, переданная при испускании (поглощении) двух фононов, $\eta\left(\omega_{q}\right)=\left(T_{1} / \omega_{q}\right) \gg 1$. Из $(3)$ и (6) следует выражение для потока энергии

$$
J_{12}(t)=-\langle\varepsilon\rangle^{t} \widetilde{\Phi}\left(N_{2}, N_{2}\right)^{t}\left\{\beta_{1}-\beta_{2}(t)\right\},
$$

где

$$
\langle\varepsilon\rangle^{t}=\lambda \bar{\varepsilon} \exp \left\{-\beta_{2}(t) \bar{\varepsilon}\right\}
$$

и вид $\bar{\varepsilon}$ зависит от формы межатомного потенциала [1].

\section{3. ПОТОК ЧАСТИЦ ЗА СЧЕТ ТЕРМИЧЕСКОЙ РЕКОМБИНАЦИИ}

В период термализации каскада процессы рекомбинации лимитируются диффузией дефектов. Поскольку выбитые атомы значительно подвижнее термодинамически равновесных вакансий, каждая вакансия представляет собой фиксированный центр, который способен захватить К Ч, движушуюся в межузельном пространстве. Вычислим $\nu-$ частоту перехода КЧ в вакансию в рамках приближения Кондона [10], когда не учитывается деформация колебательных мод, обусловленная наличием дефектов. В этом приближении допустимо дальнейшее упрощение модели, когда состояние выбитого атома, соседнего с вакансией, описывается с помощью потенциала двойной ямы. Этот потенциал имеет вид двух симметричных ям, разделенных барьером $U_{0}$, а расстояние между потенциальными минимумами $m$ и $m^{\prime}$ равно $a$. Предполагается, что $U_{0} \gg T_{1}$. Кроме того, предположим, что в подбарьерном состоянии собственные функции и собственные значения гамильтониана выбитого атома, рассматриваемого как гармонический осциллятор с частотой $\Omega$, определяются уравнением

$$
\left[\left(\frac{1}{2 M}\right) p_{x}^{2}+U(x)\right] \psi_{\lambda}(x)=E_{\lambda} \psi_{\lambda}(x) .
$$

В рамках этой модели надбарьерный переход атома $m \rightarrow m^{\prime}$ включает две стадии. На первой стадии атом в результате взаимодействия с решеткой переходит из основного состояния в возбужденное $(1 \rightarrow 2)$. На второй - теряет энергию и возвращается в потенциальную яму, а поскольку промежуточное состояние не локализовано, он может перейти как в (исходную) $m$-яму, так и в $m^{\prime}$-яму с вероятностью $1 / 2$. В результате квадрат частоты надбарьерного перехода $\nu^{2}$ включает [11] средний квадрат матричного элемента $p_{x}$ и множитель $1 / 2$,

$$
\nu^{2}=(M a)^{-2} \frac{1}{2}\left\langle\left|p_{12}\right|^{2}\right\rangle
$$

где

$$
\left\langle\left|p_{12}\right|^{2}\right\rangle=\sum_{\lambda \lambda^{\prime}} w_{\lambda}\left|\int d x \stackrel{*}{\psi_{\lambda}}(x) p_{x} \psi_{\lambda^{\prime}}(x)\right|^{2},
$$

а суммирование производится по всем начальным состояниям с весовыми множителями

$$
w_{\lambda}=\left\{2 \operatorname{sh} \frac{\xi}{2}\right\}^{-1} \exp (-\xi \lambda)
$$


и по всем конечным состояниям, удовлетворяюшим условию $E_{\lambda^{\prime}} \geqslant E_{\lambda}$. Если в качестве начального состояния принять квазиравновесие КЧ с квазитемпературой $T_{2}^{0}$, то $\xi=$ $\left(\Omega / T_{2}^{0}\right) \ll 1$. Тогда квадрат матричного элемента вычисляется методом производяшей функции [12]. Получаем

$$
\left\langle\left|p_{12}\right|^{2}\right\rangle=\frac{16}{\sqrt{\pi}} M T_{2}^{0}\left\{\sqrt{\frac{U_{0}}{T_{2}^{0}}} \exp \left[-\frac{U_{0}}{T_{2}^{0}}\right]+\frac{\sqrt{\pi}}{2} \operatorname{Erfc}\left[\sqrt{\frac{U_{0}}{T_{2}^{0}}}\right]\right\},
$$

где $\operatorname{Erfc}(x)$ - дополнительный интеграл вероятности.

Общее выражение для коэффициента скорости диффузии по вакансионному механизму Г не зависит от конкретного типа диффузанта. В приближении сильной связи оно имеет вид (см. приложение)

$$
\Gamma=2 \nu^{2} \frac{1}{\gamma(0)}
$$

где

$$
\gamma(0)=2 \pi \sum_{K K^{\prime}}\left|W_{K K^{\prime}}\right|^{2} \eta\left(\omega_{K}\right)\left[\eta\left(\omega_{K^{\prime}}\right)+1\right] \delta\left(\omega_{K}-\omega_{K^{\prime}}\right)
$$

означает затухание, входящее в двухвременную (и двухчастичную) функцию Грина в теории миграции [11], $W_{K K^{\prime}}$ - матричный элемент потенциала изотопического рассеяния фононов. Теперь, воспользовавшись формулами (8)-(10), коэффициент скорости Г можно связать с вероятностью теплового перехода КЧ в соседнюю вакансию

$$
P\left(\frac{U_{0}}{T_{2}^{0}}\right)=\frac{1}{2 \pi^{\frac{3}{2}}} \Omega\left(\frac{T_{2}^{0}}{U_{0}}\right)^{\frac{1}{2}} \exp \left\{-\frac{U_{0}}{T_{2}^{0}}\right\}
$$

А именно, справедливо соотношение

$$
\Gamma=\frac{16}{\pi} \Omega \frac{1}{\gamma(0)} P\left(\frac{U_{0}}{T_{2}^{0}}\right)
$$

Вероятность того, что в момент $t$ любое из $Z_{i}$ междоузлий, окружающих вакансию, заполнено выбитым атомом, равна $Z_{i} \widetilde{\Omega}_{\mathrm{a}} n_{V}\left(N_{2}, N_{2}\right)^{t}$, где $n_{V}$ - плотность тепловых ваканасий. Тогда поток КЧ равен

$$
J_{22}(t)=\Gamma Z_{i} \widetilde{\Omega}_{\mathrm{a}} n_{V}\left(N_{2}, N_{2}\right)^{t}=\frac{16}{\pi} \frac{1}{\gamma(0)} \Omega Z_{i} \widetilde{\Omega}_{\mathrm{a}} n_{V}\left(N_{2}, N_{2}\right)^{t} P\left(\frac{U_{0}}{T_{2}^{0}}\right)
$$

Формула (12) применима только в случае, когда нет корреляции между исходным расположением выбитых атомов и распределением вакансий. С физической точки зрения такая ситуация вполне реальна. Действительно, если КЧ первоначально имела большую энергию, то она затормаживается в области, далекой от места ее возникновения, и, следовательно, никакой пространственной коррелящии быть не может. 


\section{4. УРАВНЕНИЕ ДЛЯ КВАЗИТЕМПЕРАТУРЫ КЧ С ПОСТОЯННЫМИ КОЭФФИЦИЕНТАМИ}

Используя формулы для потока энергии $J_{12}(t)(7)$ и потока КЧ $J_{22}(t)(12)$, можно из кинетического уравнения (2) получить уравнение эволюции для обратной квазитемпературы КЧ

$$
\dot{\beta}_{2}(t)=\frac{1}{c(t)}\left\{\beta_{2}(t)\right\}^{2}\langle\varepsilon\rangle^{t}\left(N_{2}, N_{2}\right)^{t}\left\{\widetilde{\Phi}\left[\beta_{1}-\beta_{2}(t)\right]+\Gamma Z_{i} \widetilde{\Omega}_{\mathrm{a}} n_{V}\right\} .
$$

Оно одновременно описывает отвод энергии по фононному механизму (первый член) и скорость изменения энергии в каскадной зоне за счет термического отжига дефектов (второй член). В уравнение (13) вошла неравновесная теплоемкость газа КЧ

$$
c(t)=\left\{\beta_{2}(t)\right\}^{2}\left\{\left(H_{2}, H_{2}\right)^{t}-\frac{\left(H_{2}, N_{2}\right)^{t}\left(N_{2}, H_{2}\right)^{t}}{\left(N_{2}, N_{2}\right)^{t}}\right\} .
$$

Корреляционную функцию

$$
\left(N_{2}, N_{2}\right)^{t}=\int_{0}^{\varepsilon_{0}} d \varepsilon g(\varepsilon) n^{(2)}\left(\beta_{2}(t), \varepsilon\right)
$$

вычислим для случая каскада, созданного одним первично выбитым атомом. Воспользуемся выражением для плотности числа состояний КЧ, полученным в работе [1], использующей потенциал Борна-Майера,

$$
g(\varepsilon)=\frac{7}{15} \alpha\left\{\exp \left(-C_{1}^{3} \chi^{4}\right)\right\}\left\{\exp \left(3 C_{1} \chi^{2}\right)-1\right\} \nu\left(\varepsilon_{0}\right),
$$

где $\alpha=2 M R^{2}, R$ - параметр потенциала Борна-Майера, $\chi=\left(\varepsilon /\left.\right|^{*}\right)^{3 / 4}-$ безразмерная энергия КЧ, ${ }^{*}=(12)^{1 / 3} \alpha^{-1}, \nu\left(\varepsilon_{0}\right)=\varepsilon_{0} / 2 \varepsilon_{\mathrm{d}}$ - среднее число выбитых атомов, образуемых одним ПВА с энергией $\varepsilon_{0}[13], \varepsilon_{\mathrm{d}}-$ пороговая энергия смешения, $C_{1}=$ const (здесь и далее $C_{i}$ обозначают численные коэффишиенты, значения которых даны в приложении).

Интеграл (14) невозможно вычислить аналитически, так как функция $g(\varepsilon)$ имеет очень сложный вид. В связи с этим напомним одно обстоятельство. При исследовании диффузионных функций [14] и некоторых кинетических коэффициентов [15] было установлено, что форма плотности числа состояний допускает сушественные аппроксимации при условии, что аппроксимируюшая функция достаточно точно воспроизводит ту энергию, на которой расположен максимум плотности. Мы установили, что при аппроксимации плотности числа состояний $g(\varepsilon)$ гауссовой функцией

$$
\tilde{g}(\varepsilon)=\pi \alpha \nu\left(\varepsilon_{0}\right) \exp \left\{-\frac{1}{2}\left(C_{2} \chi-3\right)^{2}\right\}
$$




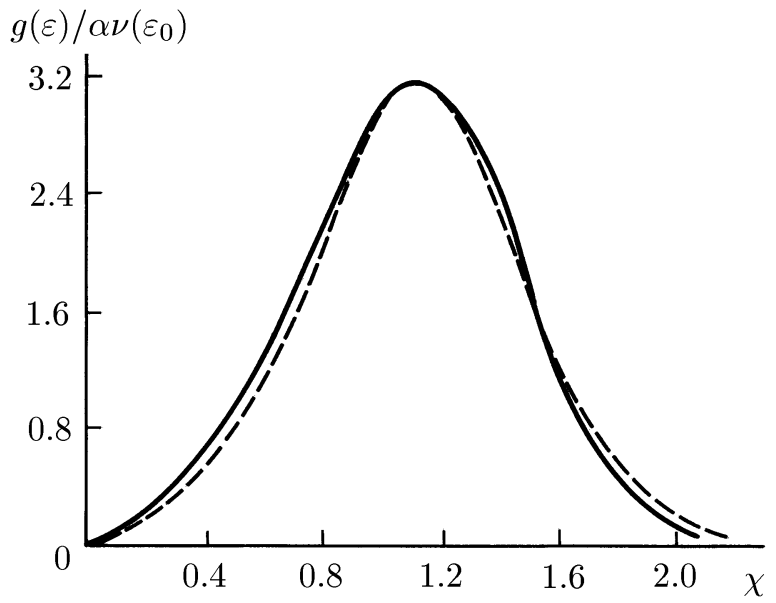

это условие выполняется.

На рисунке показаны кривые $g(\varepsilon) / \alpha \nu\left(\varepsilon_{0}\right)$ (сплошная линия) и $\tilde{g}(\varepsilon) / \alpha \nu\left(\varepsilon_{0}\right)$ (штриховая линия). Они вычислены при значениях $R=0.3 \AA$ А $\alpha=3.6$ э $\mathrm{B}^{-1}$. Подставив в (14) вместо $g(\varepsilon)$ функцию $\tilde{g}(\varepsilon)$ и вьполнив интегрирование, получим

$$
\left(N_{2}, N_{2}\right)^{t}=\lambda \nu\left(\varepsilon_{0}\right) \exp \left\{-\frac{1}{2} \beta_{2}(t)^{*} \varepsilon .\right.
$$

Теперь уравнение (13) с корреляционной функцией (15) может быть преобразовано к виду

$$
\dot{\beta}_{2}(t)=\left\{\beta_{2}(t)\right\}^{2} A(t)\left\{\beta_{1}-\beta_{2}(t)\right\}+\left\{\beta_{2}(t)\right\}^{2} B(t),
$$

где

$$
\begin{aligned}
& A(t)=\frac{1}{c(t)}\langle\varepsilon\rangle^{t} \lambda \widetilde{\Phi} \nu\left(\varepsilon_{0}\right) \exp \left\{-\frac{1}{2} \beta_{2}(t) \varepsilon\right\}, \\
& B(t)=\frac{1}{c(t)}\langle\varepsilon\rangle^{t} \lambda \Gamma Z_{i} \widetilde{\Omega}_{\mathrm{a}} \nu\left(\varepsilon_{0}\right) n_{V} \exp \left\{-\frac{1}{2} \beta_{2}(t) \varepsilon^{*}\right\} .
\end{aligned}
$$

Без дальнейших упрощений найти аналитическое решение уравнения (16) не представляется возможным. Будем пренебрегать слабой временной зависимостью коэффициентов $A(t)$ и $B(t)$, точнее, запишем их в предельном случае $\beta_{2}^{-1}(t) \rightarrow \infty$. Вьполнение такого предельного перехода [16] приводит к переходу от величин, вычисленных в рамках статистической термодинамики [9], к соответствуюшим величинам теории Линдхарда [17]. В результате получаем:

$$
\begin{gathered}
A=\lim _{\beta_{2}^{-1} \rightarrow \infty} A(t)=\frac{1}{c} \bar{\varepsilon} \widetilde{\Phi} \nu\left(\varepsilon_{0}\right)=\frac{2}{3} \bar{\varepsilon} \widetilde{\Phi}, \\
B=\lim _{\beta_{2}^{-1} \rightarrow \infty} B(t)=\frac{1}{c} \bar{\varepsilon} \Gamma Z_{i} \widetilde{\Omega}_{\mathrm{a}} \nu\left(\varepsilon_{0}\right) n_{V}=\frac{2}{3} \bar{\varepsilon} \Gamma Z_{i} \widetilde{\Omega}_{\mathrm{a}} n_{V}, \\
\bar{\varepsilon}=\lim _{\beta_{2}^{-1} \rightarrow \infty}\langle\varepsilon\rangle^{t}=\frac{\pi}{2}\left(C_{1} \alpha\right)^{-1}, \quad c=\lim _{\beta_{2}^{-1} \rightarrow \infty} c(t)=\frac{3}{2} \nu\left(\varepsilon_{0}\right) .
\end{gathered}
$$


Здесь $c$ означает теплоемкость КЧ, рассматриваемых как газ твердых сфер.

Если теперь ввести в (16) величины (17) и новую функцию

$$
u(t)=\frac{1}{\beta_{2}(t)}+a t
$$

где $a=\beta_{1} A+B$, то функция $u(t)$ будет удовлетворять нелинейному обыкновенному дифференциальному уравнению с постоянными коэффициентами

$$
\{u(t)-a t\} \dot{u}(t)+p=0 .
$$

Здесь использовано обозначение $p=-A$. Выполнив замену $\zeta=-p t$ и $u(t)=y(\zeta)$, представим последнее уравнение в виде

$$
\left\{y(\zeta)+\frac{a}{p} \zeta\right\} y^{\prime}(\zeta)=1
$$

Точное решение уравнения (18) сушествует, однако оно не может быть дано в явном виде. Действительно, получаем

$$
\zeta=C_{2} \exp \left\{\frac{a}{p} y(\zeta)\right\}-\frac{p}{a}\left\{y(\zeta)+\frac{p}{a}\right\} .
$$

Указанный недостаток решения (19) нетрудно “исправить", принимая во внимание следуюшие обстоятельства. Во-первых, термализация каскадов завершается за конечное время $t_{\mathrm{p}} \sim\left(10^{-11} \div 10^{-10}\right)$ с [8], [18]. Во-вторых, рекомбинация дефектов в каскадной зоне вносит малый вклад в общий процесс охлаждения КЧ. С учетом этого, используя решение (19), методом последовательных приближений получаем

$$
\frac{1}{\beta_{2}(t)}=\left(T_{2}^{0}-a t\right)-T_{1} \ln \left\{2\left(\frac{a+p \beta_{1}}{T_{2}^{0}-T_{1}}\right) t+1\right\},
$$

где последний член в правой части является поправкой к члену нулевого приближения. Разумеется, при записи (20) мы учитьвали конечное время термализации и начальное условие: квазитемпература КЧ равна $T_{2}^{0}$ при $t=0$.

\section{5. ВРЕМЕННОЙ ХОД КВАЗИТЕМПЕРАТУРЫ КЧ ПРИМЕНИТЕЛЬНО К АКТИВИРОВАННЫМ ПРОЦЕССАМ В ОБЛАСТИ ТЕПЛОВОГО ПИКА}

В результате бомбардировки ионами с энергией порядка нескольких МэВ в решетке возникают локальные возбуждения - тепловые пики. Физическая картина этого явления имеет большое сходство с быстрым нагревом ограниченной области твердого тела, включающей примерно $10^{3}$ атомов.

В качестве источника теплового пика (ТП) принимаются выбитые атомы с энергией порядка нескольких сотен эВ [18], [19]. В типичном ТП, точнее, внутри сферы радиуса 


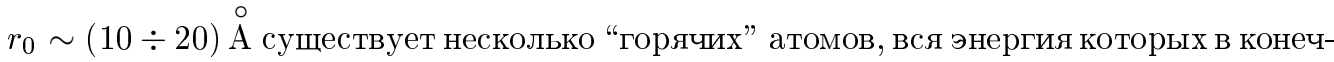
ном счете переходит в энергию колебаний решетки. В результате внутри ТП решетка разогревается до очень высоких температур, вплоть до температуры плавления. Однако благодаря быстрому подъему температуры и влиянию окружаюшей среды вешество внутри сферы радиуса $r_{0}$ остается в состоянии нагретого (или перегретого) твердого тела [18].

В феноменологической теории [18], [19] температурное поле ТП имеет вид

$$
T(r, t)=\widetilde{T}(t) \exp \left(-\frac{r^{2}}{4 D t}\right)
$$

где $D$ - коэффициент термической диффузии, $r$ - расстояние от центра сферы. Однако в работах [18], [19] используется не само поле $T(r, t)$, а только функция $\widetilde{T}(t) \sim t^{-3 / 2}$. При этом считается [8], что распределение температуры внутри сферы является пространственно-однородным. В микроскопической теории, основанной на уравнении термодинамики (2), величине $\widetilde{T}(t)$ естественно сопоставить квазитемпературу $1 / \beta_{2}(t)(20)$.

Наличие высокой температуры в области ТП стимулирует ряд активированных процессов: разупорядочение [20], осаждение [21], кристаллизацию. Кроме того, ТП может вносить дополнительный вклад в явления, обусловленные динамическими эффектами столкновений, в такие как электронные возбуждения [22], десорбция [23], термическое распыление [24]. В этой статье в качестве примера использования решения (20) рассматривается термическое распыление - испарение атомов решетки из ТП, расположенного в приповерхностном слое твердого тела. В модели Эйнштейна атом представляет собой гармонический осциллятор, колеблющийся с собственной частотой $\Omega$ в потенциальной яме глубиной $E_{\mathrm{b}}$. Предполагается, что $E_{\mathrm{b}} \gg T_{2}^{0}$. Тогда элементарный акт испарения - это переход атома с уровня основного состояния в яме на уровень поглошаюшего барьера, а энергетическая разность этих уровней $E_{\mathrm{b}}$ может быть интерпретирована как энергия связи с поверхностью. Известно, что временна́я зависимость нормальной функции распределения в гидродинамическом режиме всегда определяется зависимостью температуры от времени. Точно так же зависимость вероятности теплового перехода (11) от времени полностью определяется временны́м ходом квазитемпературы (20). Тогда

$$
P\left(\beta_{2}(t)\right)=\left(2 \pi^{\frac{3}{2}}\right)^{-1} \Omega\left(E_{\mathrm{b}} \beta_{2}(t)\right)^{-\frac{1}{2}} \exp \left\{-\left(E_{\mathrm{b}} \beta_{2}(t)\right)\right\} .
$$

Коэффициент термического распыления $S_{\text {therm }}$ характеризует число испущенных атомов, приходящихся на один налетающий ион. Если поток испарившихся атомов через свободную поверхность записать в форме [25] $j(t)=N_{1} r_{\mathrm{s}} P\left(\beta_{2}(t)\right)$, то коэффициент распыления имеет вид

$$
S_{\text {therm }}=\Pi\left(E_{0}\right) \int_{0}^{t_{\mathrm{p}}} d t j(t)=\left(2 \pi^{\frac{3}{2}}\right)^{-1} N_{1} r_{\mathrm{s}} \Pi\left(E_{0}\right) \Omega E_{\mathrm{b}} \frac{1}{\widetilde{K}} \Gamma\left(-\frac{3}{2}, \frac{E_{\mathrm{b}}}{T_{2}^{0}}\right) .
$$

В формуле (21) использованы следуюшие обозначения: $E_{0}$ - энергия падающего иона; $(4 \pi / 3) r_{\mathrm{s}}^{3}=1 / N_{1} ; \quad \Pi\left(E_{0}\right)=\pi r_{0}^{3} / \lambda\left(E_{0}\right)$ - средняя величина нагретой площади, приходящейся на один падаюший ион, в условиях хаотического падения пучка $[26] ; \lambda\left(E_{0}\right)=$ 
$\left\{\sigma\left(E_{0}\right) N_{1}\right\}^{-1}$ - длина свободного пробега бомбардируюших ионов; $\sigma\left(E_{0}\right)$ - сечение рассеяния; $\Gamma(\alpha, x)$ - неполная гамма-функция. Последнее равенство в (21) получено в пределе $t_{\mathrm{p}} \rightarrow \infty$, поскольку величина интеграла в (21) в основном формируется на нижнем пределе. Что касается величины $\widetilde{K}$, также вошедшей в $(21)$, то ее удается вычислить в том же приближении, которое использовалось при выводе (20), т.е. пренебрегая малым вкладом рекомбинации. В области высоких температур основной вклад дает двухфононный процесс. Тогда, учитывая (6), (17) и (20), получаем

$$
\widetilde{K}=\frac{2}{3} \beta_{1} \bar{\varepsilon} \widetilde{\Phi}=C_{3} \widetilde{\Omega}_{\mathrm{a}} \bar{\varepsilon} \frac{1}{M^{2}} \int d q q^{6} \Delta_{q}\left[\frac{1}{\omega_{q}^{3}}|V(q)|^{2}\right]^{2} .
$$

В феноменологической теории активированных процессов [18], [19], [24] вводится дополнительная физическая величина $n_{j}$ - число реализаций активированного процесса за время жизни ТП. Эта величина связана с количеством тепла $Q_{2}$, высвободившимся внутри сферы радиуса $r_{0}$, соотношением [19]

$$
n_{j}=\frac{1}{\pi^{2}}\left(\frac{Q_{2}}{E_{\mathrm{b}}}\right)^{\frac{2}{3}} \tilde{\rho}
$$

Предварительно выразив энергию связи через упругую постоянную $\bar{g}[11], \quad E_{\mathrm{b}}=$ $\left(1 / \pi^{2}\right) \bar{g} r_{s}^{2}$, запишем величину $\tilde{\rho}$ в принятых нами обозначениях

$$
\tilde{\rho}=\Omega r_{\mathrm{s}}^{2}\left(\frac{\widetilde{K}}{\bar{g}}\right)^{-1} .
$$

Используя (20) и (22), получаем искомое соотношение

$$
S_{\text {therm }}=C_{4} N_{1} r_{\mathrm{s}} \Pi\left(E_{0}\right)\left\{\nu\left(\varepsilon_{0}\right)\right\}^{-\frac{2}{3}} f_{1}\left(\frac{E_{\mathrm{b}}}{T_{2}^{0}}\right) n_{j} .
$$

Оно связывает коэффициент распыления с числом реализаций $n_{j}$ и функцией, зависяшей от начальной квазитемпературы ТП,

$$
f_{1}\left(\frac{E_{\mathrm{b}}}{T_{2}^{0}}\right)=\left(\frac{E_{\mathrm{b}}}{T_{2}^{0}}\right)^{-\frac{11}{6}} \exp \left(-\frac{E_{\mathrm{b}}}{T_{2}^{0}}\right)
$$

\section{6. ОБСУЖДЕНИЕ РЕЗУЛЬТАТОВ}

6.1. Два подхода в теории термализации. При решении задачи термализации каскадов в твердых телах возможны два направления исследования: феноменологический подход [18], [19], основанный на уравнении теплопроводности, и подход, предложенный в данной работе, в рамках которого система описывается на термодинамическом уровне с использованием представлений микроскопической теории.

Основная трудность феноменологической теории заключается не столько в отыскании решения ее основного уравнения, сколько в применимости самой концепции теплопроводности. Дело в том, что в качестве источника теплового пика рассматриваются 
выбитые атомы, причем энергия, которую они передают решетке, высвобождается в небольшом объеме среды. Можно ли в таком случае считать, что линейное уравнение теплопроводности, которое описывает распространение тепла в так называемой непрерывной модели, применимо в этих условиях? Тем более, что наличие процессов, протекающих в широком интервале температур, казалось бы неминуемо должно приводить к квазилинейному уравнению теплопроводности.

Однако это еше не все. Чтобы достигнуть более заметного спада температуры, в стандартном решении уравнения теплопроводности отбрасывается множитель $\exp \left\{-\left(r^{2} / 4 D t\right)\right\}$. Теперь временна́я эволюция температуры каскада определяется функцией $\widetilde{T}(t) \sim t^{-3 / 2}$, которая имеет сингулярность при $t=0$. Чтобы "исправить" ситуацию, предлагается [19] либо ввести стартовое время $t_{0}=r_{\mathrm{s}}^{2} / 4 D$ и использовать решение вида [26] $\left(t+t_{0}\right)^{-3 / 2}$, либо ограничить во всех интегральных преобразованиях нижний предел. Все это вносит в теорию дополнительную неясность.

Представленная вьше термодинамическая теория не подтверждает постулаты работ [18], [19], [24]. Во-первых, на основе системы уравнений для термодинамических параметров (1) установлено, что квазитемпература КЧ удовлетворяет уравнению Абеля первого рода (18), а не линейному уравнению параболического типа. Во-вторых, найдено точное решение (19) уравнения Абеля. Показано, что в условиях слабой рекомбинации спад квазитемпературы (20) близок к линейному, а отклонение от линейности описывается слабоменяюшейся (логарифмической) функцией. Решение в виде алгебраических функций, которое используется в [18], [19], [24], не найдено.

Кинетический подход к изучению неравновесных явлений в период термализации представляется более глубоким. Он может давать обоснование приближений, используемых при более формальной постановке проблемы, в частности, в ее феноменологической трактовке.

6.2. Кинетика термического распыления. Как видно из соотношений (22), (23), коэффициент распыления пропорционален $\tilde{\rho}$. Аналогом величины $\tilde{\rho}$ в феноменологической теории [18], [19] является отношение атомной диффузионной способности к термической диффузионной способности решетки, которое в ряде случаев, например, при изучении радиационного разупорядочения сплавов, может считаться равным единице [18], [19]. Для того чтобы воспользоваться представлениями химической кинетики, упростим нашу задачу, предполагая $\tilde{\rho}=1$. Тогда остается только одно взаимодействие бомбардирующего иона с атомом решетки, а, следовательно, и одно сечение рассеяния $\sigma\left(E_{0}\right)$. В этом случае интересующая нас "квазихимическая" реакция описывает преврашение одних частиц в другие:

$$
A_{1}+S\left\{A_{2}\right\} \stackrel{k\left(1 / \beta_{2}\right)}{\longrightarrow} \text { распыленные атомы, }
$$

где $A_{1}$ - бомбардирующий ион, $S\left\{A_{2}\right\}$ - совокупность атомов решетки в области ТП, $k\left(1 / \beta_{2}\right)$ - коэффициент скорости термического распыления. Принимая во внимание (22), (23), находим

$$
S_{\text {therm }} \sim k\left(T_{2}^{0}\right), \quad k\left(T_{2}^{0}\right)=\widetilde{B}\left(\frac{T_{2}^{0}}{E_{\mathrm{b}}}\right)^{\frac{5}{2}} \exp \left\{-\frac{E_{\mathrm{b}}}{T_{2}^{0}}\right\}
$$


(выражения для $S_{\text {therm }}$ и $\widetilde{B}$ в частном случае рассеяния по законам твердых шаров даны в приложении). Сравнивая (24) с модифицированным уравнением для реакции с энергией активации $E_{\mathrm{b}}$ в форме Аррениуса [27]

$$
k_{n}(T)=\widetilde{B}\left(\frac{T}{E_{\mathrm{b}}}\right)^{n} \exp \left\{-\frac{E_{\mathrm{b}}}{T}\right\},
$$

видим, что формулы совпадают при $n=5 / 2$ и $T=T_{2}^{0}$.

Вблизи равновесия сложных систем аррениусова кинетика хорошо изучена [27]. Однако в условиях ТП, когда имеется разрыв термодинамических параметров кристалла и подсистемы КЧ, теоретическое понимание кинетики на столь же высоком уровне отсутствует. Поэтому обоснование модифицированной кинетики Аррениуса следует считать основным результатом приложения теории термализации, точнее, температурного хода (20) к активированному процессу - термическому распылению. Отметим также, что другими примерами реализации модифицированной кинетики могут быть бимолекулярные химические реакции в газовой фазе, когда временна́я эволюция системы (типа плазмы) обусловлена бинарными столкновениями [27].

6.3. Краткое заключение. В данной работе, посвященной исследованию термализации каскада смешений в твердом теле, получены следуюшие результаты:

1. Квазитемпература КЧ удовлетворяет уравнению Абеля первого рода (18), которое одновременно описьвает отвод энергии по фононному механизму и изменение энергии внутри каскадной зоны за счет термической рекомбинации дефектов.

2. Точное решение уравнения (18) может быть дано только в неявном виде. Однако в условиях слабой рекомбинации спад квазитемпературы КЧ в период термализации аппроксимируется функцией (20), близкой к линейно спадающей функции.

3. Временна́я зависимость квазитемпературы КЧ (20) использована для анализа активированного процесса - распыления атомов в приповерхностном слое. Показано, что коэффициент скорости этого процесса описывается модифицированным выражением Аррениуса $k_{n}\left(T_{2}^{0}\right)$ с показателем степени $n=5 / 2$.

\section{ПРИЛОЖЕНИЕ}

Из-за математических трудностей не будем рассматривать диффузию атома в реальных кристаллах по вакансионному механизму, а ограничимся обсуждением упрощенной модели [28]. В качестве такой модели принимается цепочка атомов с одной вакансией. Она может использоваться и в задаче о каскаде смешений, если принять, что вокруг вакансии находится только один выбитый атом. Предполагается также, что на стадии термализации, после того как выбитьй атом затормозился, его скорость достаточно мала и энергия меньше высоты потенциального барьера вблизи вакантного узла. В противном случае никакого захвата атома вакансией не произойдет.

Введение гамильтониана миграции $T_{A}[28]$ в полный гамильтониан системы дает возможность исследовать диффузионную подвижность в рамках теории линейной реакции [9]. В этом случае коэффициент скорости диффузии Г определяется соотношением

$$
n_{V}\left(\frac{a^{2}}{2}\right) \Gamma=\int_{0}^{\infty} d t\langle\dot{d} \dot{d}(-t)\rangle_{0} \exp (-\varepsilon t), \quad \varepsilon \rightarrow+0 .
$$


Здесь $\dot{d}=i\left[T_{A}, d\right]$ - скорость мигрирующего атома, $d$-его дипольный момент. Записав $\dot{d}$ в операторах Паули $\stackrel{+}{a}_{m}, a_{m}$ [11], имеем

$$
\dot{d}=\frac{1}{2} a \nu \sum_{m}\left(\rho_{m}+\stackrel{+}{\rho}_{m}\right),
$$

где $\rho_{m}=\stackrel{+}{a}_{m+1} a_{m}$, и для коэффициента переноса получаем

$$
n_{V} \Gamma=\nu^{2} \sum_{m m^{\prime}} \int d t\left\langle\stackrel{+}{\rho}_{m^{\prime}} \rho_{m}(-t)\right\rangle_{0} \exp (-\varepsilon t) .
$$

Используя спектральную теорему, выразим коррелящионную функцию в (П.1) через спектральную интенсивность $J_{m m^{\prime}}$. Тогда

$$
n_{V} \Gamma=\nu^{2} \sum_{m m^{\prime}} \int d \omega J_{m m^{\prime}}(\omega) \int_{0}^{\infty} d t^{\prime} \exp \left(i \omega t^{\prime}-\varepsilon t^{\prime}\right) .
$$

Спектральная интенсивность $J_{m m^{\prime}}$, в свою очередь, может быть выражена через массовый оператор $M(\omega)$ и затухание $\gamma(\omega)$ двухвременной (и двухчастичной) функции Грина

$$
J_{m m^{\prime}}(\omega)=\frac{1}{\pi} F(\omega)\left[\exp \left(\beta_{1} \omega\right)+1\right]^{-1}\left(n_{m}+n_{m+1}\right) \delta_{m m^{\prime}},
$$

где

$$
F(\omega)=\gamma(\omega)\left[(\omega-M(\omega))^{2}+\gamma^{2}(\omega)\right]^{-1} .
$$

Здесь $n_{m}$ - собственные значения оператора плотности вакансий [28]. Отметим также, что функция Грина построена на основе антикоммутатора. Теперь, подставляя (П.3) в (П.2), в предельном случае сильного взаимодействия $\gamma^{2}(0) \gg \omega_{k}^{2}$ получаем искомое соотношение (10).

В заключение запишем в полном виде коэффициент распыления (24)

$$
S_{\text {therm }}=C_{5} N_{1} r_{0}^{3} \frac{r_{\mathrm{s}}}{v\left(E_{0}\right)} k\left(T_{2}^{0}\right) .
$$

Предэкспоненциальный множитель $\widetilde{B}$ в выражении для $k\left(T_{2}^{0}\right)$ пропорционален сечению упругого рассеяния $\sigma\left(E_{0}\right)$. Поэтому явный вид $\widetilde{B}$ зависит от модели атом-атомного потенциала и интервала энергий, к которому относится энергия налетающего иона $E_{0}$. В случае облучения кристалла тяжелыми ионами $\left(\mathrm{Ar}^{+}, \mathrm{Xe}^{+}\right)$с энергией в интервале $\left(10 \div 10^{2}\right)$ КэВ сечение рассеяния можно вычислить в приближении твердых шаров. В этом приближении имеем

$$
\widetilde{B}=\frac{1}{2} a_{\mathrm{B}}^{2} N_{1} \frac{E_{\mathrm{R}}}{E_{0}^{\frac{1}{2}}} \frac{Z_{0} Z}{\left(Z_{0}^{\frac{2}{3}}+Z^{\frac{2}{3}}\right)^{\frac{1}{2}}} \frac{M_{0}+M}{M M_{0}^{\frac{1}{2}}},
$$

где $a_{\mathrm{B}}$ - радиус Бора, $Z_{0}$ и $M_{0}$ - заряд и масса бомбардируюшего иона, $Z$ - заряд атома решетки, $E_{\mathrm{R}}$ - постоянная Ридберга.

Ниже приведены значения численных коэффициентов $C_{i}$, вошедших в некоторые из представленных в статье формул.

$$
C_{1}=\frac{4}{7}=0.57 ; \quad C_{2}=2.6 ; \quad C_{3}=9 \cdot 10^{-2} ; \quad C_{4}=4 \cdot 10^{-2} ; \quad C_{5}=3 \cdot 10^{-2} .
$$




\section{Список литературы}

[1] Ю. А. Кашлев. ТМФ. 2000. Т. 123. № 3. С. 485.

[2] Ю. А. Кашлев. ТМФ. 2001. Т. 126. № 2. С. 311.

[3] W. Brandt. Appl. Phys. 1974. V. 5. № 1. P. 1.

[4] Y. Jkari. J. Phys. Soc. Japan. 1979. V. 46. P. 97.

[5] T. Mc. Mullen. J. Phys. 1977. V. 7. P. 2041.

[6] A. P. Mills (Jr.), L. Pfeiffer. Phys. Rev. Lett. 1979. V. 43. P. 1961.

[7] W. Prandt, R. Paulin. Phys. Rev. B. 1972. V. 5. P. 2430.

[8] M. W. Thompson, R.S. Nelson. Phil. Mag. 1962. V. 7. № 84. P. 2015.

[9] Д. Н. Зубарев. Неравновесная статистическая термодинамика. М.: Наука, 1971.

[10] M. Lagos. Solid State Comm. 1984. V. 50. № 8. P. 777; 1989. V. 69. № 1. P. 11.

[11] Ю. А. Кашлев. ТМФ. 1975. Т. 24. № 2. С. 265.

[12] R. Kubo. Phys. Rev. 1952. V. 86. № 6. P. 929.

[13] Г. Н. Кинчин, Р. С. Пиз. УФН. 1956. Т. 60. № 4. С. 590.

[14] D. K. Brice, B. L. Doyle. J. Nucl. Mat. 1984. V. 120. № 2. P. 230.

[15] Ю. А. Кашлев. ТМФ. 1998. Т. 116. № 3. С. 442.

[16] Ю. А. Кашлев, Н. М. Садыков. ТМФ. 1997. Т. 111. № 3. С. 483.

[17] Й. Линдхард. УФН. 1969. Т. 99. Вып. 2. С. 249.

[18] Д. Динс, Д. Винйард. Радиационные эффекты в твердых телах. М.: ИЛ, 1960.

[19] F. Seits, J. S. Kochler. Solid State Physics. 1956. V. 2. P. 305.

[20] H. Blank. Phys. Stat. Sol. 1972. V. 10(a). P. 465.

[21] R. Chang. Acta Metallur. 1957. V. 5. P. 257.

[22] C. A. Andersen, J. R. Hinthorne. Annal. Chem. 1973. V. 45. P. 1421.

[23] G. H. Vineyard. Rad. Effects. 1976. V. 29. P. 245.

[24] R. Kelly. Rad. Effects. 1977. V. 32. P. 91.

[25] G. M. Pound. J. Phys. Chem. Ref. Data. 1972. V. 1. P. 135.

[26] R.S. Nelson. Phil. Mag. 1965. V. 11. № 110. P. 291.

[27] В. Штиллер. Уравнение Аррениуса и неравновесная кинетика. М.: Мир, 2000.

[28] L. P. Kadanoff, J. Swift. Phys. Rev. 1968. V. 168. № 3. P. 310.

Поступила в редакцию 22.III.2001 г., после доработки 7.VI.2001 г. 